\title{
A QoS-aware network supporting an E-learning framework
}

\author{
Antonio Pescapè ${ }^{1,2}$, Stefano Russo ${ }^{1,2}$, Vincenzo Vecchio ${ }^{1,2}$, Giorgio Ventre ${ }^{1,2}$ \\ 'Università degli Studi di Napoli 'Federico II'- DIS, Dipartimento di Informatica e Sistemistica, \\ Napoli, \\ ${ }_{2}^{2}$ ITEM - Laboratorio Nazionale CINI per l'Informatica e la Telematica Multimediali, Napoli
}

\begin{abstract}
Distance Learning has largely benefited from the incredible progress that internetworking technologies have made in the last years. The provision of multimedia services with guaranteed QoS is currently an important research issue in computer engineering, especially in the networking and information management areas. In this paper, we present an Italian research structure (ITEM - CINI of Naples) created to grant remote access to real laboratories and to provide distance learning services such as discovery and delivery of multimedia resources in the educational application domain. In the first part of the paper we present the structure and the activities of the ITEM laboratory and in the second part, metadata models for Quality of Software (QoS)-aware information management systems are presented.
\end{abstract}

Key words: Remote experiences, Metadata, Quality of Service, Standards, Profiles.

\section{INTRODUCTION}

Distance learning has become a powerful mechanism, giving the possibility to reach learners with a time and space independent approach. New technologies, such as e-mail, chat, video-conferences, have improved the ability to overcome obstacles related to physical distance. Virtual laboratories have gained incredible success in the last years, offering the possibility to give access to structures that are usually meant for an intrainstitution kind of use. Anyway, simulation is only an initial stage for these

The original version of this chapter was revised: The copyright line was incorrect. This has been corrected. The Erratum to this chapter is available at DOI: 10.1007/978-0-387-35700-3_33 
types of experiments but does not offer the same opportunities that real laboratories may offer. For some disciplines, such as physics and electrical and mechanical engineering, the answers provided by real instruments represent a crucial aspect for the entire learning process. On the other hand, many of the existing tools for distance learning are in fact software applications for simulations or, in other words, 'virtual laboratories', which hamper the possibility of observing what really happens during a laboratory experiment. Furthermore, the design of simulation software largely depends on how the student's perception is understood by the software designer. In some cases the procedures necessary to run the system may go beyond the student's knowledge background. Virtual experiments may also lead to assumptions that lead to erroneous results, but in spite of this, students' learning processes may depend on the quality of the software used more than on the actual intellectual capabilities. Moreover, 'virtual' experiments may reduce interest and curiosity hampering the ability to acquire new knowledge and leading sometimes to hurried results following some predefined steps. The scenario described above deprives students from many of the concepts that are derived from experiments, reducing the opportunity to ask questions such as 'what happens if...'. A small amount of original ideas will be produced and it is likely that learners familiar with the software will obtain better results. In this sense we think that virtual laboratories play an important role in the distance learning process, but not as a replacement to real laboratories. The ITEM laboratory has set up a rather complex structure that involves students' individual feelings helping the overall learning process. A very important aspect is reality: students are no longer observers but actors within the experiments. All this is possible only when the network infrastructure is able to guarantee some specific QoS requirements.

In this paper we present a generic model for QoS-aware Information Management Systems, able to support a guarantee of the QoS in search and delivery of information objects over a network infrastructure (such as the Internet and/or Intranet). The model identifies two kinds of objects, actors and involved components, and their roles. The metadata models we analyze are specialized for the e-learning domain. For this application domain, we also propose QoS extensions to standard metadata models for profiling users, information services and resources, capable of supporting learning resources discovery and personalized delivery with QoS guarantee. The ITEM Lab structures offers the possibility of performing remote experiments together with the opportunity to receive multimedia content, creating in this way a complete and integrated learning experience. 
Modern web-based Information Management Systems usually provide users with two major functionalities, namely information retrieval and delivery. Two major problems that new systems have to deal with are related to the overwhelming increase of digital information being produced, which hampers the retrieval of the relevant information, and to the assurance of the quality of the service to the end user during e-learning and remote experiences activities. QoS is actually one of the more active research areas in the field of computer networking. The basic concept is that networks should be able to offer communication services with adequate performance, i.e. suited to both the characteristics of the data to be transmitted, and to the requirements of the users. During the past few years, several models for QoS aware network have been proposed. In the majority of these proposals, the user is seen as responsible for negotiating with the network for the service characteristics of its communications. The idea is that users interact with QoS aware network devices in order to book resources such as link capacity or memory buffers, so that the traffic they generate can receive guarantees of the values of some performance parameters such as communication delay, loss rate, etc. This approach comes from the assumption that the user of a communication service is the one who knows the requirements that a certain application or service has in terms of end-to-end communication performance. Unfortunately, this approach suffers from the problem of how to provide the users with this QoS related information, so that network resources can be allocated efficiently and, possibly, at the lowest cost for the users themselves. In fact, this information is influenced by a large number of factors: the characteristics of the media and of the content, the existing relationships with other media, the service models adopted in the network infrastructure, the type and requirements of the applications, and, finally, the end user needs. Another issue is related to how the user can avoid dealing with all the technical aspects of resource reservation, and with the possible changes that these technicalities can have with the evolution in network models and technologies. In order to automate QoS provisioning, we claim that QoS metadata should be linked to the media to be delivered and, similarly to the retrieval period, to the user preferences, making these accessible directly to the user applications. 


\section{ITEM LAB AND LABNET PROJECTS}

ITEM (Laboratorio Nazionale per l'Informatica e la TElematica Multimediali) is the National Laboratory for Computer Science and Multimedia Systems. It was founded at the beginning of 2000 as an effort of CINI. CINI (Consorzio Interuniversitario Nazionale per l'Informatica) is a consortium of Computer Science and Computer Engineering research units operating within some Italian Universities. It was built in 1990 as a nonprofit organisation to promote the cooperation of Italian computer scientists and engineers in nation-wide and international projects in the area of ICT. ITEM is already involved in several research projects related to multimedia communications and e-Learning applications. In particular, it is involved in several national research projects in the area of remote access to network infrastructures and laboratories (LABNET), and to an IST Program project in the area of QoS over IP networks (CADENUS). ITEM includes three research units: Distributed Computing Architectures, Network Infrastructures, Multimedia Applications and Services (Pescapé et al, 2001 p179) Labnet projects aim to improve the learning skills both of students (master degree and undergraduate school) and employees of enterprises (small, medium and large). Such a wide range of opportunities is meant to produce a flexible learning structure. Even though Universities and schools are used to experimental activities on concrete and complex devices, they usually have problems in the field of purchase and management costs as well as in the lack of staff able to use this infrastructure. Both small and medium enterprises and big corporate can experiment this kind of difficulty. One of Labnet's main goal is to make students manage real laboratories (centralized or distributed), remotely accessible, providing them with the necessary infrastructure. Other educational benefits of this approach rely on the possibility of having students work together on a virtual platform so that they can talk about the current experiment and results. Complex equipment (radio communication devices, industrial robot, network and TLC equipments, electronic and electrical measuring equipments, biomedical devices) are remotely controlled by students located on several sites, while the supervisor supports all activities concerning the remote experiment and follows each step of the trial. The first step toward the ultimate realization of the Laboratory is the release of a prototype useful for application testing. In fact, common goal of Labnet projects is the realization of an IDS (Integrated Didactic System) able to make remote resources accessible to both teachers and students located in several sites thanks to interfaces and integration of systems and multimedia technology. Labnet projects are 
structured in order to satisfy operating IDS needs and to solve e-learning technological and methodological problems. This objective is obtained both by the integration of existing technologies based on heterogeneous and complex systems (in the short period) and by the development of innovative technologies and implementation of specific prototypes (in the middle period). Therefore, the integrated Labnet projects represent an innovative solution in the context of interactive and remote learning on real laboratories in the Telecommunications and Multimedia area.

To realise the IDS, the CINI consortium planned four types of actions:

- ITEM Laboratory for Multimedia technologies and Communications issues;

- Design and implementation of network architecture for access to remote labs;

- Design and implementation of network architecture for classrooms (University and School) connection;

- Restructuring legacy research laboratory in order to include it in the Labnet network.

To achieve the scientific and technological goals, the following aspects will be developed:

- Use of infrastructures realized during the activities listed above; eLearning applications;

- Design and implementation of tools meant for controlling Measuring equipments;

- Design and implementation of tools meant for controlling Robot equipment;

- A framework in order to test the tools developed in the field (University, School and Corporate).

Taking into account the realisation of an infrastructure to access remote laboratories the open issues depends mainly on the lack of interoperability between the legacy software applications and the measuring equipment in order to obtain a real remote control on the device involved in the experiment.

Another problem to solve is the interoperability between different software applications working to exchange data on a certain number of heterogenous network platforms. Labnet projects aim to realize a platform (hardware and software) capable of solving these limitations derived from actual constraints and therefore platform and network independent Services 
(Pescapé et al, 2001 p188). ITEM Lab has mainly focused on Applied Physics (Atomic, Molecular and Optical Physics and Superconductor Science and Technology), Electronic and Electrical Measuring and Robotic Control. In the Electronic and Electrical Measuring field Labnet projects have two key objectives: the implementation of a 'Tele-didactic' system and the realization of 'Tele-measuring' platform, both supported by video streamed lessons. In the first case the software architecture uses a web application in order to show the possible experience and a client-server application control to manage the measuring. In the second case it is possible to measure the precision and the accuracy of a device, connecting the device to the Labnet network makes it possible to calibrate the device. In order to realize Physics experiments ITEM Lab currently collaborates with LAFIDIN (LAboratorio FIsico DIdattico a Ingegneria) lab of the University 'Federico II' of Napoli. LAFIDIN lab makes available 25 measuring stations with educational science equipment. While in the University there are many Physics labs, in most Secondary schools there is a lack of these labs and of technical operators. The cooperation between ITEM and LAFIDIN and the realization of a remote laboratory gives a chance of overcoming these difficulties.

To avoid the drawbacks of 'virtual experiences' ITEM Lab has achieved a Network and Multimedia infrastructure able to satisfy all requirements related to multimedia issues: storage, management, processing and streaming of multimedia contents. In this context a 'Remote Desk' has been designed and implemented to allow recording and real time streaming of lessons and seminars from ITEM site. The 'Remote Desk' configuration is simple and automatic in order to have this configuration used by non-expert teachers too. The 'Remote Desk' schema is based on a hardware-software system in which video, audio, slides and documents (experiment videos or simulation) can be automatically integrated. Video contents are made using a remotely controlled digital video camera with semiautomatic tracking. Multimedia documents are stored on a server farm (for asynchronous access) and transmitted on the 'LABNET channel'. These results have been obtained thanks to the integration of architectures developed at the University of Napoli. In particular, two Video on Demand applications (named MuSA (Canonico, 1999) and DiVa (Canonico, 1998)) have been customized in order to allow, respectively, the storage on a server cluster and the streaming with VCR-like functionalities. Research is now focused on support for Quality of Service and in the next sections we describe metadata models for QoS-aware Information Management Systems (IMS, 2001). 

MANAGEMENT SYSTEMS

In the networking research field, the term Quality of Service is used to define the delivery of an agreed, defined and measurable level of service to a user when trying to access a digital resource. Traditionally, the term QoS has been associated with the correct delivery of digital resources over a computer network and many approaches have been followed which range from the planning of QoS-aware networking technologies to QoS-aware communication protocols based on the Internet Protocol IP. The Best-Effort service provided by the latter has shown its limitations over the years, even though it is currently the most popular service. Current QoS models over IP networks are the IETF Integrated Services (Braden, 1994, 1997), and Differentiated Services (Blake 1998, Zhang 2001). Both architectures have limitations and a number of solutions have been explored that combine the best of both approaches overcoming the limitations of either when applied alone. By QoS-metadata of digital resources we mean a description of their Quality-of-Service requirements, for their proper delivery and utilization by users. QoS requirements of a given resource are either intrinsic to the nature of the resource itself (e.g., an audio resource may be suitable for hi-fi quality production), or they are related to the way the resource should be delivered to the user (e.g., bandwidth requirements for a guaranteed realtime delivery). We also agree with the recent view that QoS should not only be related to network performance to assure the correct delivery of a digital resource or a service, but also and not least to meeting user requirements. The key issue is where these resource description parameters (metadata) reside and how they are managed (Bouch, 2000). In principle, three approaches may be envisaged. One is to manage no metadata at all: this is the current approach for most web-based Information Management Systems; this maps naturally to a best-effort delivery model of current large network infrastructures such as the Internet. A second approach is to mandate the presence of metadata for each resource, and to wire them tightly with the resource itself; an example of this is the emerging MPEG-7 standard for audiovisual resources, which provides a rich set of standardized tools to describe multimedia content (MPEG, 2001). A third 'intermediate' approach is to keep a description of resources logically separate from them, so as to have complementary information about resources, that can be exploited by information management tools for providing extended or to improve the quality of the service delivered. Our reference framework takes this latter approach. The model identifies actors and components involved, and their 
role, in a very generic QoS-oriented Information Management Systems, able to support services for both discovery and delivery of multimedia resources over an open network infrastructure.

QoS-metadata is needed at two levels:

- User Preferences: these are stored in the user profile and contain metadata describing the user access devices both in terms of hardware an software. This category should also contain choices of personal and device preferences.

- Content QoS: this metadata describes the information object to be delivered, such as the object format, the connection required, the hardware or software requirements, etc

Support for Quality of Service (QoS) metadata in standards seems to be minimal and restricted to software and platform requirements. Although this is adequate for search and discovery purposes, which are based on user profile preferences and Service Provider offerings, it is far from adequate for QoS network and content delivery purposes. Managing and matching the above described profiles and metadata are described in the architecture presented here, which can be considered general and applicable to any type of digital resources. Care has been taken that all the QoS related metadata fields are clearly separated from the rest of the data models and could be as easily incorporated in alternative data models. The steps of the interactions between the various architectural components in a search and delivery scenario are the following:

1. User logs onto the system. At this stage a broker retrieves the user's profile that will be used in the search/delivery process;

2. Some session related information has to be present in the system. Even if QoS parameters are stored somewhere in the user profile, the user has to confirm them;

3. User performs a query. At search time, QoS awareness of the broker will retrieve only information objects that are actually accessible through the device in use, meaning that search results will include only information objects that match user preferences both cognitive and technical, and content preferences. On the other hand, if the user expressly asks for all the available content, the broker will retrieve all the Information Objects in a given domain, regardless of what kind of device he is using to access the system;

4. The broker returns a list of available resources to the user. Since all the information objects are fully described by their metadata the user can now make an informed choice of what he thinks may be relevant; 
5. The user picks one resource from the list. Before this resource can be delivered QoS metadata will have to be retrieved from the broker. This metadata will be used to negotiate the quality of service with the network and assure a correct delivery of the Information Object itself. At this stage the user has chosen what he thinks is a relevant resource, which is suited for the access device in use. In order to assure the correct delivery it is necessary that the resource will receive appropriate QoS provision on the end-to-end delivery network. An example is represented by those services where the user is prompted with a list of the different digital formats the desired resource is available in. The selection of the digital document is manually made by the user;

6. By checking the content QoS Metadata the user application checks if the QoS requirements can be met. In our proposed, we include a simple resource characterization that would allow a QoS aware user client to reserve the required network resources. If the user application cannot reserve the necessary network resources the user is notified and another resource may be chosen instead or the user can compromise the expected quality;

7. The user retrieves the chosen resource with the required QoS;

8. The user logs out and the profile is saved to be used on a following session.

\section{CONCLUSIONS}

We believe that metadata for supporting delivery of digital resource with a predefined quality of service, and for a better integration of the retrieval and delivery tasks, will be an integral part of future Information Management Systems. In this paper we have presented a generic model of advanced distributed information management systems developed during our research activity. The presented solution is based on the usage of standard metadata for supporting information discovery and delivery. The adoption of standard models will allow interoperability among future systems. However, current standards metadata models do not explicitly address QoS issues as we have shown in the educational domain. We have also proposed to extend standard metadata models for digital resources, the user profiles and finally the information services, by including ad hoc categories encompassing the QoS parameters needed for applications to be able to support resource discovery and delivery with guarantee of the quality 
of service provided. The ITEM research described in this paper has been partially evaluated during the GUARDIANS project, a European Union 5th Framework Programme project. This approach has allowed more sophisticated and productive searches than current web-based resource discovery systems provide. During the delivery phase, the QoS-aware information management system built during the GUARDIANS project, has used the extra QoS-metadata to negotiate the quality-of-service with network equipment, according to advanced QoS network models and protocols.

\section{REFERENCES}

Blake S. et al (1998) An Architecture for differentiated Services. RFC2475.

Bouch A., Sasse M. A. and Demeer H. (2000) Of packets and people: a user-centered approach to quality of service. Quality of Service, IWQOS. 2000 Eighth International Workshop.

Braden R. et al (1994) Integrated Services in the Internet Architecture: an Overview. RFC 1633.

Braden R. et al (1997) Resource ReSerVation Protocol (RSVP) -- Version 1 Functional Spec. RFC2205.

Canonico R., Capuozzo G., Iannello G. and Ventre G.(1999) MuSA: a scalable multimedia server based on clusters of SMPs, First Workshop on Cluster Computing at the ACM International Conference on Supercomputing

Canonico R., Caretti G., Fadini B. and Ventre G.(1998) DiVA: un'Architettura Eterogenea per Servizi di Video Broadcasting Cooperativo, AICA98, Annual Conference

IMS (2001) Instructional Management Systems, Global Learning Consortium, http://www.imsproject.org/index.html [accessed 12 Nov 2001]

MPEG (2001) Overview of the MPEG-7 Standard, http://mpeg.telecomitalialab.com/standards/mpeg-7/mpeg-7.htm [accessed 28 Nov 2001].

Pescapè A, Ventre G., Delfino F. and Fadini B (2001) Un laboratorio per applicazioni di rete, di calcolo distribuito e multimediali: il laboratorio ITeM del CINI (Consorzio Interuniversitario Nazionale per l'Informatica), Didamatica2001, Annual Conference - vol 2, pps. 179-187.

Pescapè A., Ventre G., Tamasi G., Luponio C., Fadini B. (2001) Esperienze di didattica remota su laboratori: i progetti LABNET2 del CINI, Didamatica2001, Annual Conference - vol 2, pps. 188-199.

Zhang G. and Mouftah H. T. (2001) End-to-end QoS guarantees over Diffserv networks, 2001. Proceedings of the Sixth IEEE Symposium on Computers and Communications. IEEE Comput. Soc, Los Alamitos, xvi+737 pp.p.302-9. 